\title{
ESTABILIDAD FÍSICO-QUímICA DE LAS SEMILLAS DESHIDRATADAS DE CASTAÑA (Bertholletia excelsa)
}

\author{
Victor SOTERO ${ }^{1}$, Ursula MONTEIRO ${ }^{1}$, Claudia MERINO-ZEGARRA ${ }^{1}$, Martha MACO ${ }^{1}$, Ericka DAVILA ${ }^{2}$, Dora \\ GARCIA $^{2}$
}

1 Instituto de Investigaciones de la Amazonía Peruana (IIAP). Laboratorio de Sustancias Naturales Bioactivas (LSNB).Carretera Iquitos-Nauta, km 4.5, Iquitos, Perú. e-mail: vsotero@iiap.org.pe

2 Universidad Nacional de la Amazonía Peruana (UNAP). Facultad de Ingeniería Química. Departamento de Química. Freyre 616. Iquitos, Perú.

\section{RESUMEN}

Se trabajó con semillas de castaña Bertholletia excelsa, procedentes de dos regiones amazónicas del Perú: Loreto (Tamshiyacu) y Madre de Dios (Puerto Maldonado), estas muestras fueron liofilizadas y sometidas a diferentes concentraciones de actividad de agua a temperatura ambiente, para verificar la estabilidad de sus componentes a los cambios de humedad relativa. Es importante notar que sus isotermas, ajustadas con la ecuación de B.E.T., presentaron una buena estabilidad en el rango de 0.1 a 0.7 . las mayores concentraciones en sodio, y selenio con 422.50 y $32.17 \mathrm{mg} / 100 \mathrm{~g}$ respectivamente, se encontraron en la harina liofilizada de Puerto Maldonado, y potasio, magnesio, calcio, zinc, hierro, manganeso y cobre con $2156.43 \mathrm{mg} / 100 \mathrm{~g}, 671.40,36.77,7.12,6.19,4.10$ y 3.53 $\mathrm{mg} / 100 \mathrm{~g}$ respectivamente en la harina liofilizada de Tamshiyacu.

PALABRAS CLAVE: castaña, deshidratación, adsorción.

\section{PHYSICAL AND CHEMICAL STABILITY OF DEHYDRATED SEEDS OF BRAZIL NUT (Bertholletia excelsa)}

\section{ABSTRACT}

Brazil nuts Bertholletia excelsa were collected from two Amazon regions of Peru, Loreto (Tamshiyacu) and Madre de Dios (Puerto Maldonado), to obtain freeze-dried flour to verify the stability of its components to changes in relative humidity and temperature. The isotherms of the samples, adjusted by the B.E.T equation, were stable to activity of water ranking from 0.1 to 0.7 ). The highest concentration of sodium and selenium (422.50 and 32.17 $\mathrm{mg} / 100 \mathrm{~g}$ respectively) were in freeze-dried flour from Puerto Maldonado, while the samples from Tamshiyacu were higher in potassium, magnesium, calcium, zinc, iron, manganese and copper $(2156,43,671,40,36,77,7,12$, $6,19,4,10$ and $3,53 \mathrm{mg} / 100 \mathrm{~g}$ respectively).

KEYWORDS: Brazil nut, dehydratation, adsorption. 


\section{INTRODUCCIÓN}

La castaña (Berthellotia excelsa), es un arbol nativo del Suramerica, distribuida en la cuenca masónica de Bolivia, Brasil, Colombia, Perú, guyana y Venezuela. Es una planta social y se la encuentra en determinadas zonas formando los denominados castañales (Pechnik et al., 1950; Flores, 1997).

La semilla del fruto maduro es comestible, se consume cruda, tostada, salada y en la preparación de dulces y productos de confitería, es altamente calóricA debido a la alta concentración de lípidos y proteína (Tabla 1), aunque sea deficiente en lisina, Isoleucina y treonina. Es una excelente fuente de aminoácidos sulfurados, metionina y cisteína como se observa en la Tabla 2 (Pechnik et al., 1950; Elias \& Bressani, 1961; Pereira, 1976; Regitano D'Arce, 1998). La castaña es uno de los pocos frutos con altos valores en selenio, según Chang et al. (1995) obtuvo valores de 0.03 a 512 ppm de este elemento con muestras frescas de distintas regiones de la amazonía brasileña. La presencia de selenio en la dieta ha sido asociada con la protección contra el desarrollo de tumores en estudios realizados con animales de laboratorio, específicamente en la inhibición del cáncer de mama (Chang et al., 1995; Ip \& Lisk, 1994).

El liofilizado es una operación utilizada para la eliminación del agua mediante desecación al vacío y a muy bajas temperaturas. Utilizado principalmente en la industria alimentaria y farmacéutica, aunque también se puede utilizar para fabricar materiales como el aerogel (Brenna et al., 1998).

La actividad de agua $\left(\mathrm{a}_{\mathrm{w}}\right)$, es un parámetro que indica la disponibilidad de agua en un alimento para que existan reacciones químicas, bioquímicas (p.e. oxidación de lípidos, reacciones enzimáticos, reacción de Maillard) y desarrollo microbiano. Este es un parámetro usado como indicador para predecir la vida útil de un alimento. La actividad de agua $\left(\mathrm{a}_{\mathrm{w}}\right)$ del medio representa la fracción molar de las moléculas de agua totales que están disponibles, y es igual a la relación que existe entre la presión de vapor de la solución respecto a la del agua pura (p/po). El valor mínimo de $\mathrm{a}_{\mathrm{w}}$ en el cual las bacterias pueden crecer varía ampliamente, pero el valor óptimo para muchas especies es mayor a 0.99. Algunas bacterias halófilas (bacterias que se desarrollan en altas concentraciones de sal) crecen mejor con $\mathrm{a}_{\mathrm{w}}=0.80$ (U. Navarra, 2009).

Por tanto, la isoterma de un producto relaciona gráficamente, a una temperatura constante, el contenido en humedad de equilibrio de un producto con la actividad termodinámica del agua del mismo, ya que en el equilibrio, este último parámetro es igual a la humedad relativa del aire que rodea al producto. Las isotermas son útiles para el análisis y diseño de varios procesos de transformación de alimentos, tales como secado, mezcla y envasado de los mismos. Son además son importantes para predecir los cambios en la estabilidad de los alimentos y en la elección del material de empaque adecuado. Existen diversos estudios en cuanto a las características de secado, isotermas de adsorcion y deserción, como los realizados en maíz por Doymaz \& Pala (2003), de hortalizas, por García et al. (2007) y de cereales para desayuno (Prieto et al., 2006) y de haba (MatosChamorro \& Rajo-Angulo, 2010).

La obtención y modelado de la isoterma de adsorción de agua de la harina de castaña, es de suma importancia para la determinación de materiales de embalaje, condiciones de almacenamiento, evaluación de mezclado con otras harinas u otros alimentos en polvo; para predecir su vida útil y para la determinación de condiciones óptimas de secado (Chirife \& Iglesias, 1978).

Varias ecuaciones empíricas y semiempíricas se han propuesto para correlacionar el contenido de humedad de equilibrio con la actividad de agua de un alimento, sin embargo, la ecuación de G.A.B (GuggenheimAnderson-de Boer) es de amplio uso en alimentos y es recomendada por el proyecto Europeo COST 90, que trata sobre propiedades físicas en alimentos (Wolf et al.,1984). Esta ecuación está basada en la teoría de adsorción de B.E.T. (Brunauer-Emmet-Teller), la cual da una explicación física a los parámetros involucrados en ella. Otro factor importante, a la hora de elegir la ecuación a utilizar, es la simplicidad y el tiempo que pueda necesitar el cálculo de la humedad de equilibrio (Mclaughlin \& Magee, 1998).

El objetivo de este trabajo fue determinar experimentalmente las isotermas de adsorción de humedad de la harina liofilizada de nueces de castaña, de dos procedencias del Perú a la temperatura ambiente de la ciudad de Iquitos $\left(27^{\circ} \mathrm{C}\right)$, y modelarlas aplicando la ecuación propuesta por B.E.T.

\section{MATERIAL Y MÉTODOS}

\section{MATERIAL BIOLÓGICO}

Se utilizó como materias primas semillas de castaña colectados de los distritos de Tamshiyacu en la provincia de Maynas (Loreto), y ciudad de Puerto Maldonado en la provincia de Tambopata (Madre de Dios), en las fechas de enero a marzo de 2009.

\section{PARTE EXPERIMENTAL}

Las semillas de castaña, fueron sometidas a prensado en la empresa D'Souza (Iquitos), de donde se extrajo el aceite y la torta residual, fue liofilizada en un equipo de Laboratorio del Instituto de Medicina Tradicional de 
EsSalud de Loreto. Para esto la muestras fueron previamente congeladas a $-20{ }^{\circ} \mathrm{C}$ y posteriormente sometidas a liofilizado a 100 mtorr por $12 \mathrm{hrs}$.

\section{MÉTODOS ANALÍTICOS}

Los análisis bromatológicos se realizaron utilizando la metodología dada por Adolfo Lutz (1985) y los elementos se determinaron por espectrofotometría de absorción atómica. (Osborne \& Vgogt, 1978).

Para las Isotermas de adsorción, se utilizó el método estático, que consistió en colocar la muestra en un desecador con una solución saturada de un compuesto de humedad relativa conocida, se utilizaron los siguientes compuestos: $\mathrm{NaOH}, \mathrm{CH}_{3} \mathrm{COOK}, \mathrm{MgCl}_{2}$, $\mathrm{NaCl}$ y $\mathrm{KOH}$, para mantener actividades de agua de $0.083,0.113,0.225,0.328,0.753$ y 0.83 a temperatura ambiente, después de obtener peso constante a los seis días, se obtuvo la humedad ganada por la muestra y con estos datos se encontró la actividad de agua de cada muestra. Para obtener el modelo de la curva se ajusta de acuerdo a la ecuación de B.E.T. (1938), que se define así:

$$
\begin{aligned}
& \text { aw } /\left(\mathrm{m}(1-\mathrm{aw})=1 / \mathrm{m}_{0}+\mathrm{C}+\left[(\mathrm{C}-1)\left(\mathrm{m}_{0}-\mathrm{C}\right)\right] \mathrm{aw}\right. \\
& \text { Donde } \\
& \text { aw }: \text { actividad de agua } \\
& \mathrm{m}: \text { humedad ganada durante la adsorción } \\
& \mathrm{m}_{0} \text { : contenido de humedad en la monocapa } \\
& \mathrm{C} \text { : constantes. } \\
& \text { Esta ecuación se grafica como una correlación } \\
& \text { lineal de la siguiente manera: } \\
& \mathrm{Y}=\mathrm{a}+\mathrm{bX}
\end{aligned}
$$

\section{RESULTADOS Y DISCUSIÓN}

El rendimiento de la extracción de aceite de castaña de dos procedencias del Perú, de Puerto Maldonado y de Tamshiyacu, se presenta en la Tabla 3. Se observa que las muestras de Puerto Maldonado son de mayor peso que las de Tamshiyacu, y dejan menos residuo, como torta.

De acuerdo a los análisis bromatológicos de las harinas de castaña de dos procedencias, antes y después de liofilizar (Tabla 4), la diferencia es notable en el incremento de la concentración de proteína en ambos productos finales.

Los resultados de los análisis de los elementos que contienen la fracción mineral de las castañas antes y después de ser liofilizadas, se presentan en la Tabla 5. Se observa que la castaña, en este caso prensada o liofilizada, es una importante fuente de elementos destacando nítidamente el potasio con 1957.97 a $2156.43 \mathrm{mg} / 100 \mathrm{~g}$ magnesio con 593.85 a 676.80 y sodio de 236.17 a $675.0 \mathrm{mg} / 100 \mathrm{~g}$. Cabe notar que la liofilización en sí no incrementa mayormente la concentración de elementos, ya que en algunos casos tiende a disminuir.

De acuerdo a la Figura 1, donde se grafica la isoterma de adsorción de la harina de castaña liofilizada a diferentes concentraciones de actividad de agua a la temperatura de $27^{\circ} \mathrm{C}$. Las isotermas obtenidas tanto para las muestras de Tamshiyacu como las de Puerto Maldonado, son muy parecidas, tanto que se superponen, no presentando una diferencia marcada. El comportamiento de concavidad es bastante típico par este tipo de muestras sobre todo de frutos tropicales. Se nota una baja concentración de humedad en la monocapa de tal modo a que esto no permitirá la oxidación de lípidos, pardeamiento enzimático o actividad enzimática (Hubinger et al., 1992; Gabas et al., 1999). Comparando con la isoterma de la nuez de Arachis hipogea, realizada por Benedetti \& Jorge (1987), presentan las curvas de humedad de equilibrio de la nuez seca, la cual presenta valores menores de humedad de equilibrio hasta un nivel de $70 \%$ de humedad relativa (\%) o sea aw de 0.7 , para el caso de las castañas estas presentan valores similares también hasta esta humedad.

En las figuras 2 y 3 , se presentan las isotermas de adsorción ajustada a la ecuación de B.E.T para harina liofilizada de castaña de Tamshiyacu y Puerto Maldonado a $27^{\circ} \mathrm{C}$. La ecuación de sorción de B.E.T representa una base de interpretación de isotermas multicapas desorción y ha sido aplicada en adsorción de gases y vapores en superficies y sólidos porosos, como también en absorción de vapor, especialmente agua, por polímeros y otros materiales homogéneos (Zug, 2002) 
FOLIA ESTABILIDAD FÍSICO-QUÍMICA DE LAS SEMILLAS DESHIDRATADAS

Tabla 1. Composición centesimal de las semillas deshidratadas de la castaña (Bertholletia excelsa).

\begin{tabular}{lcccc}
\hline $\begin{array}{c}\text { COMPONENTE } \\
(\mathbf{g} / \mathbf{1 0 0 g})\end{array}$ & $\begin{array}{c}\text { Pechnik et al. } \\
(\mathbf{1 9 5 0 )}\end{array}$ & $\begin{array}{c}\text { Elias \& Bressani } \\
(\mathbf{1 9 6 1 )}\end{array}$ & $\begin{array}{c}\text { Pereira } \\
(\mathbf{1 9 7 6 )}\end{array}$ & $\begin{array}{c}\text { Regitano D'Arce } \\
(\mathbf{1 9 9 8 )}\end{array}$ \\
\hline Humedad & 3.01 & 2.0 & 2.41 & 4.81 \\
Proteína & 6.46 & 16.3 & 5.85 & 9.6 \\
Lípidos & 9.3 & 68.3 & 65.45 & 67.8 \\
Fibras & 4.6 & 2.6 & - & - \\
Carbohidratos & 3.2 & - & - & 4.13 \\
Minerales & 3.5 & - & - & 3.65 \\
\hline
\end{tabular}

Tabla 2. Composición de los aminoácidos de la harina de castaña (mg/100 g de proteína).

\begin{tabular}{lccc}
\hline AMINOÁCIDO & $\begin{array}{c}\text { Zucas et al. } \\
(\mathbf{1 9 7 5 )}\end{array}$ & $\begin{array}{c}\text { Antunes \& Markakis } \\
(\mathbf{1 9 7 7 )}\end{array}$ & $\begin{array}{c}\text { Glória } \\
\text { (1977) }\end{array}$ \\
\hline Isoleucina & 3280 & 3644 & 3750 \\
Leucina & 7450 & 7234 & 8710 \\
\hline Lisina & 3390 & 3296 & 3710 \\
\hline Metionina & 4560 & 6176 & 9550 \\
Fenilalanina & 4010 & 4544 & 4920 \\
\hline Treonina & 2890 & 2800 & 3160 \\
Valina & 4100 & 5552 & 5920 \\
Cisteína & 11780 & -- & 4120 \\
\hline
\end{tabular}

Tabla 3. Rendimiento de la extracción de aceite de castaña de dos procedencias por prensado.

\begin{tabular}{lcc}
\hline \multicolumn{1}{c}{ PARÁMETRO } & PTO. MALDONADO & TAMSHIYACU \\
\hline Peso (Kg) & 4450 & 1350 \\
\% Aceite & 2839.5 & 699 \\
Torta (harina) & 1610.5 & 651 \\
\% extracción aceite & 63.81 & 51.78 \\
\% extracción torta & 36.19 & 48.22 \\
\hline
\end{tabular}


Tabla 4. Análisis bromatológico (\%) de la harina de castaña antes y después de liofilizado.

\begin{tabular}{lrcrr}
\hline \multirow{2}{*}{ PARÁMETRO } & \multicolumn{2}{c}{ SIN LIOFILIZAR } & \multicolumn{2}{c}{ LIOFILIZADAS } \\
\cline { 2 - 5 } & TAMSHIYACU & \multicolumn{1}{c}{ PTO. MALDONADO } & \multicolumn{1}{c}{ TAMSHIYACU } & PTO. MALDONAD0 \\
\hline Minerales & $7.69 \pm 0.03$ & $7.18 \pm 0.02$ & $8.11 \pm 0.03$ & $7.46 \pm 0.03$ \\
Aceite & $35.14 \pm 0.11$ & $35.88 \pm 0.82$ & $33.39 \pm 0.79$ & $34.71 \pm 0.89$ \\
Proteína & $36.119 \pm 0.71$ & $38.95 \pm 1.05$ & $51.18 \pm 2.7$ & $49.66 \pm 0.8$ \\
Carbohidratos & 15.251 & 12.73 & 6.17 & 6.98 \\
\hline
\end{tabular}

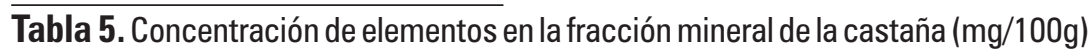

\begin{tabular}{|c|c|c|c|c|}
\hline \multirow{3}{*}{ MINERALES } & \multicolumn{4}{|c|}{ CASTAÑA (mg/100g) } \\
\hline & \multicolumn{2}{|c|}{ SIN LIOFILIZAR } & \multicolumn{2}{|c|}{ LIOFILIZADAS } \\
\hline & HMD & HT & HLMD & HLT \\
\hline$K$ & $1957.97 \pm 276.50$ & $1973.77 \pm 119.28$ & $1438.27 \pm 82.67$ & $2156.43 \pm 72.32$ \\
\hline Mg & $676.80 \pm 28.31$ & $635.65 \pm 21.26$ & $593.85 \pm 7.75$ & $671.40 \pm 41.20$ \\
\hline $\mathrm{Na}$ & $675.00 \pm 55.68$ & $491.00 \pm 29.82$ & $422.50 \pm 25.20$ & $236.17 \pm 8.95$ \\
\hline Se & $38.67 \pm 2.56$ & $27.00 \pm 2.17$ & $32.17 \pm 1.75$ & $23.00 \pm 1.80$ \\
\hline $\mathrm{Ca}$ & $34.27 \pm 0.20$ & $37.41 \pm 0.60$ & $31.83 \pm 1.15$ & $36.77 \pm 0.13$ \\
\hline Zn & $6.12 \pm 0.23$ & $6.93 \pm 0.15$ & $5.69 \pm 0.28$ & $7.12 \pm 0.07$ \\
\hline Fe & $5.59 \pm 0.25$ & $5.88 \pm 0.24$ & $5.18 \pm 0.15$ & $6.19 \pm 0.24$ \\
\hline Mn & $3.11 \pm 0.06$ & $3.77 \pm 0.10$ & $3.04 \pm 0.11$ & $4.10 \pm 0.32$ \\
\hline Cu & $3.29 \pm 0.02$ & $3.59 \pm 0.06$ & $3.11 \pm 0.09$ & $3.53 \pm 0.01$ \\
\hline
\end{tabular}

Leyenda:

HMD: harina de castaña desengrasada de Puerto Maldonado.

HT: harina de castaña desengrasada de Tamshiyacu.

HLMD: harina de castaña liofilizada de Pto Maldonado.

HLT: harina de castaña liofilizada de Tamshiyacu. 
FOLIA
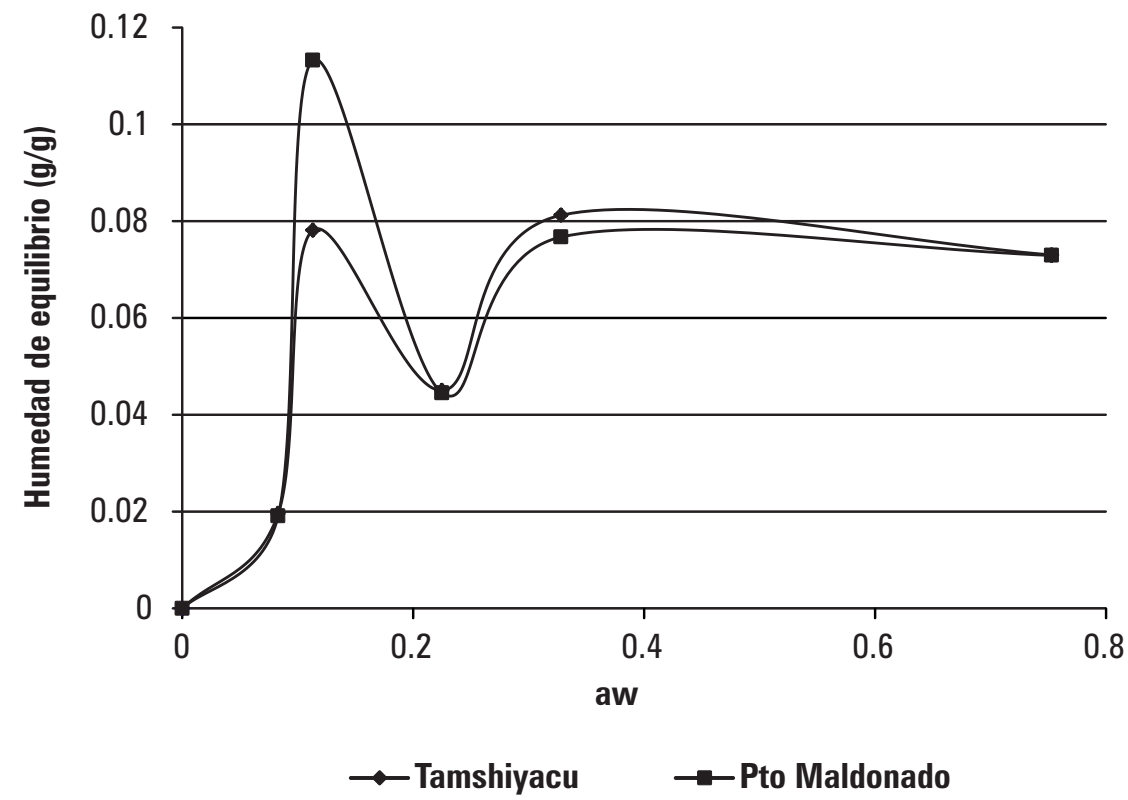

Figura 1. Isoterma de absorción de harina de castaña liofilizada.

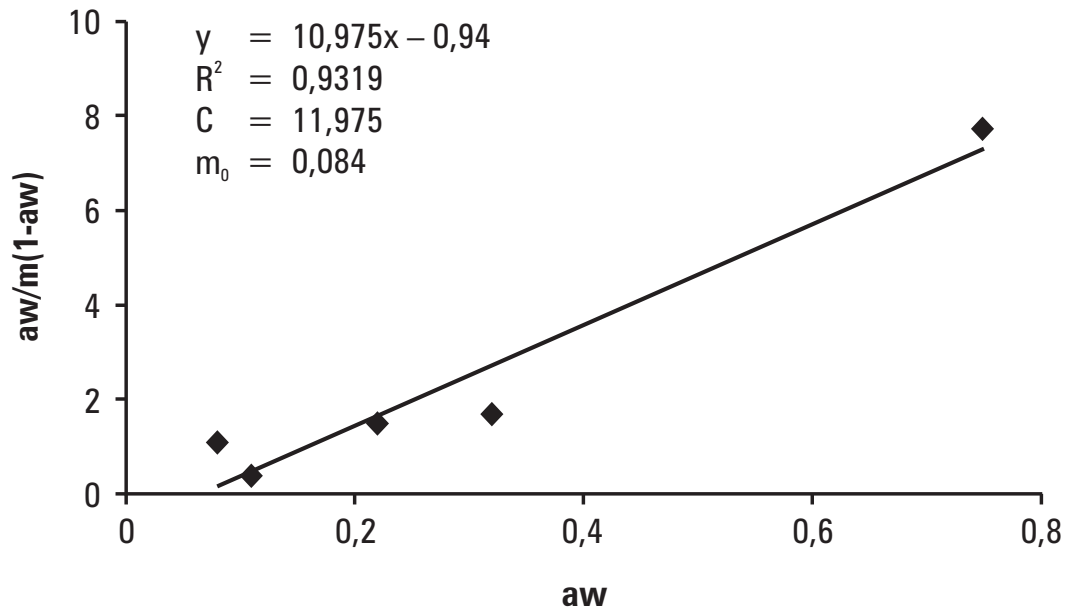

Figura 2. Isoterma ajustada a la ecuación de B.E.T para harina de castaña liofilizada de Tamshiyacu. 


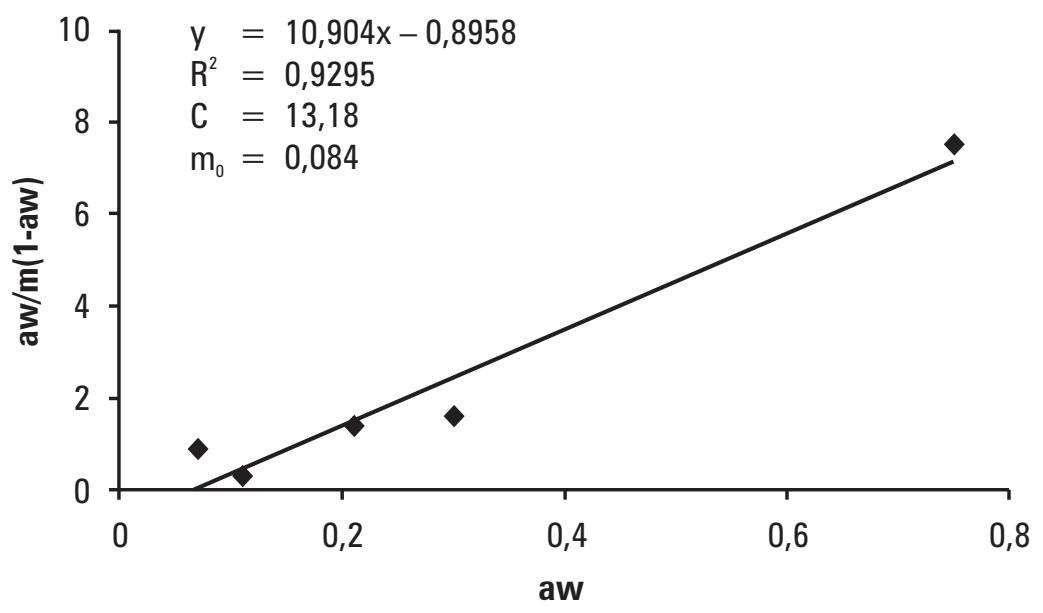

Figura 3. Isoterma ajustada a la ecuación de B.E.T para harina de castaña liofilizada de Puerto Maldonado.

\section{BIBLIOGRAFIA CITADA}

Adolfo Lutz, 1985. Normas analíticas do Instituto Adolfo Lutz. Métodos químicos e físicos para analise de alimentos. 2 ed. São Paulo. Vol 1, 583 p.

Benedetti, B. C.; Jorge, J. T. 1987. Curvas de umidade de equilibrio de varios grãos. Cienc. Tecnol. Aliment. Vol 7(2): 172-188.

B. E. T.; Brunauer, S. ; Emmett, P. H. ; and Teller, E. 1938. Adsorption of gases in multimolecular layers. J. Am.Chem. Soc. 60:309-314.

Brennan, J.G.; Butters, J.R.,Cowell, N.D.; Lilley, A.E.V. Las Operaciones de la Ingenieria de los Alimentos. Zaragoza. Editorial Acribia. p. 412417. 1998.

Antunes, A. J.; Markakis, P. 1977. Protein suplementation of navy beans with Brazil nuts, $J$. Agric. Food Chem. Vol.25 (5):1096-1098.

Chang, J.C.; Gutenmann W.H.; Reid, C.M.; Lisk, D.J. 1995. Selenium content of Brazil nuts from two geographic locations in Brazil, Chemosphere. Vol. 30,(4):801-802.

Chirife, J.; Iglesias, H. A. 1978. Equations for fitting water sorption isotherm of foods: Part I: a review. Journal of Food Technology, Vol. 3: 159-174.

Doymaz, I.; Pala, M. 2003. The thin - layer drying characteristics of corn. Journal of Food Engineering. Vol. 60(2):125-130.

Elias, L. G.; Bressani, R. 1961. The nutritive value of Brazil nut oil. J. Am Oil Chem. Soc. Vol. 38: 450.
Flores,S. 1997. Cultivo de frutales nativos amazónicos: Manual para el extensionista. Lima: Mirigraf S.R.L. 63-69 p.

Gabas, A. L.; Telis-Romero, J.; Menegalli, F. C. 1999. Thermodynamic models for water sorption by grape skin and pulp. Drying Technology. p.17.

Garica, S.V.; Schmalku, M.C.; Tanzariello, A.; 2007. Isoterma De adsorcion y cinetica de sacado de ciertas hortalizas y aromaticos cultivados en Misiones. RIA. Vol. 36(1): 115-129.

Gloria, M. M. 1997. Obtenção e caracterização de concentrado e isolado protéico de torta de castanha-do pará. Universidade de Sao Paulo. Piracicaba. Dissertação de Mestrado. 72 p.

Ip, C.; Lisk, D.J. 1994.Characterization of tissue selenium profiles and antecarcinogenic responses in rats fed natural sources of selenium-rich products. Carcinogenesis. Vol.15 (4):573-576.

Matos-Chamorro, M.; Raho-angulo, R. 2010. Influencia del tamaño de partícula en la isoterma de adsorción del harina de haba (Vicia faba l), Rev. Investig. Ciencia. Tecnol. Aliment. Vol. 1(1): 5157.

Mclaughlin, C. P.; Magee, T. R. A. 1998. The Determination of Sorption Isotherm and the Isosteric Heats of Sorption for Potatoes. Journal of Food Engineering. Vol. 35 (3): 267-280.

Osborne, D.R.; Vgogt, P. 1986. Análisis de los nutrientes de los alimentos. Zaragoza. Ed. Acribia S.A. 258 p. 
Pechnik, E.; Borges, P.; Siqueira, R. 1950. Estudo sobre a castanha do Pará. Arquivos Brasileiros de Nutrição. Vol. 7(1):7-42.

Pereira, P. L. 1976. Extratos solúveis da castanha-dobrasil (Bertholletia excelsa, H.B.K.), Campinas. Dissertação de Mestrado. Faculdade de Engenharia de Alimentos- Universidade Estadual de Campinas. $43 \mathrm{p}$.

Prieto, F.; Gordillo, A.; Prieto, J.; Gomez, C.; Roman, A. 2006. Evaluacion de las isotermas de adsorcion de cereales para desayuno. Superficies y Vacio. Vol. 19(1): 12-19.

Regitano D'Arce, R. M. A. B.; Siqueira, F. M. 1995. Obtenção do leite e farinhas de castanha do Pará. In: Congresso e Exposição Latinoamericana sobre Processamento de Óleos e Gorduras. Campinas, Anais. Sociedade Brasileira de Óleos e Gorduras. p. 265-267.

Wolf, W.; Spiess, W. E. L.; Jung, G.; Weisser, H.; Bizot, H.; Duckworth, R. B. 1984. The watervapour sorption isotherms of microcrystalline cellulose and purified potato starch. Results of collaborative study. Journal of Food Engineering. Vol. 3(1): 51-73.

Zucas, S. M.; Silva, E. C. V.; Fernandez, M. I. 1975. Farinha de castanha do Pará. Valor de sua proteina. Rev. Farm. Bioquím. Univ. S. Paulo. Vol. 13(1,): 133-144.

Hubinger, M.; Menegalli, F. C.; Aguerre, R. J.; Suarez, C. 1992. Water adsorption isotherms of guava, mango and pineapple. J. Food Sci. Vol. 57: 1405.

Zug, J. P. 2002. Isotermas de Sorción de Tres Etapas y Modelos de Sorción Restringida Monografía Nro.6. Universidad de Buenos Aires. 15p.

Pagina web consultada:

http://www.unavarra.es/genmic/curso\%20microbiolo gia\%20general/notas_de_microbiologia_de_los_al.h tm 